\title{
Unmoderated Posters Infections and Inflammatory Disease
}

\author{
UP-59 \\ Infectious Complications of Trans-rectal Ultrasound Guided \\ Biopsy of the Prostate: A Single Institution Experience \\ Kovac, Evan; Al-Qaoud, Talal; Baillargeon, Emilie; Artho, Giovanni; \\ Aprikian, Armen \\ McGill University Health Centre, Montreal, QC, Canada \\ Introduction and Objectives: Trans-rectal ultrasound (TRUS) guided biopsy \\ of the prostate is a commonly performed procedure to confirm the diag- \\ nosis of adenocarcinoma. Antibiotic prophylaxis with a fluoroquinolone \\ is recommended to prevent the seeding of rectal flora into the urinary \\ tract. However, infectious complications of the procedure are a growing \\ concern, owing to the emergence of fluoroquinolone resistant bacteria. \\ Our Objective is to evaluate the incidence of fluoroquinolone-resistant \\ bacteremia after TRUS-guided biopsy of the prostate in a tertiary care centre. \\ Methods: We retrospectively reviewed all TRUS-guided biopsies of the \\ prostate from January 1-December 31, 2010 at a single tertiary care hos- \\ pital radiology department. A standard 3-day course of oral Ciprofloxacin \\ prophylaxis was used for all patients, beginning 1 day prior to the biopsy.
}

A systematic chart review was conducted to identify patients that presented to our centre within 30 days of biopsy and their respective culture results. In addition, each patient was contacted by phone and administered a questionnaire to determine whether the patient presented post-biopsy to a hospital other than ours.

Results: Of the 207 patients who underwent TRUS biopsy, 133 (65\%) were included in the study. We identified 2 patients with fluoroquinolone-resistant bacteremia $(1.5 \%)$ and another 3 with a positive urine culture $(2.3 \%)$. 1 patient developed C-difficile associated diarrhea $(0.8 \%)$. There were no mortalities and the average hospital stay was 2.4 days.

Conclusions: Our retrospective review identified patients presenting with fluoroquinolone-resistant bacteremia and/or positive urine cultures postTRUS guided biopsy of the prostate. Using a 3-day course of fluoroquinolone prophylaxis, we found that our infectious complication rate is similar to that previously published. It is possible that this rate would be slightly different had we been able to contact all patients undergoing biopsy. 